\title{
MONITORING ODOUR EMISSSIONS FROM AN OIL \& GAS PLANT: ELECTRONIC NOSE PERFORMANCE TESTING IN THE FIELD
}

\author{
Laura Capelli*(laura.capelli@polimi.it), Selena Sironi \\ Politecnico di Milano, Department of Chemistry, Materials, and Chemical Engineering "Giulio Natta"
}

\begin{abstract}
This paper focuses on performance testing of electronic noses for environmental odour monitoring in terms of their capability of correctly classifying odours at low odour concentrations. The studied case concerns the realization of an electronic nose network for the continuous monitoring of odour emissions from a crude oil extraction and separation plant. The novelty of the work consists in the fact that performance testing, which is typically carried out in laboratory before installation in the field for environmental odour monitoring outside the plant boundaries, in this case was carried out after installation with the aim of testing the instruments performances in the effective working conditions. This involved the necessity to develop a specific and repeatable procedure to obtain samples at known quality and concentration in the field. Electronic nose performance was evaluated in terms of classification accuracy, which produced satisfactory results towards the considered olfactory classes.
\end{abstract}

Index Terms - continuous odour monitoring, electronic nose, performance testing, odour concentration, sample preparation

\section{INTRODUCTION}

Air quality monitoring for the detection and recognition of environmental odours is an important field of application for electronic noses, and it has become more and more important over the last decade due to the broadened concept of air quality, which has led odours to be included as a form of air pollution [1]. Actually, odour is one of the main cause of citizens' complaints to the environmental authorities, especially in highly populated regions, where residential units often are found close to industrial areas. In this scenario, electronic noses are particularly interesting because while other better-established and solid analytical techniques can be used to detect specific compounds, electronic noses are currently the only method capable of quantifying and classifying odours in real time [2].

At present, most regulation approaches are based on a first step of odour emission quantification through the measurement of odour concentration by dynamic olfactometry [3], followed by exposure evaluation by dispersion modelling [1]. However, there are several situations for which dispersion modelling is hardly applicable, due to the difficulty of assessing a representative odour emission rate, the variability of emissions or the presence of multiple sources. In such cases it might be useful to get free from the necessity of minutely characterizing the emission, and to determine the exposure to odours directly where their presence is lamented. Electronic noses could be used for this purpose [4].

Despite a wide range of objective criticalities connected to the use of electronic noses for environmental odour monitoring in ambient air, such as drift, cross-sensitivity to atmospheric conditions or low sensitivity, there are some examples of successful applications of trained systems of gas sensors [2,5]. Based on this experience, it was decided to realize a network of 8 electronic noses for the continuous monitoring of the odour emissions from a crude oil extraction and separation plant.

Because of the above mentioned criticalities associated with this kind of application that are connected mainly (but not only) to the difficulty of obtaining stable responses with varying external conditions (e.g. humidity and temperature) [2], it was decided to carry out an extensive evaluation of the electronic nose network performances after the installation in the field. The aim of the performance testing was to verify that the satisfactory results obtained during the instrument training and performance testing in the laboratory prior to installation were achievable and reproducible outdoors in the field, which is all but obvious. Another difficulty is represented by the fact that due to the distance between the location of the electronic noses and the lab, target odour samples for the instrument performance testing had to be prepared directly on site. For this reason, one innovative aspect of this work consists in the development of a specific procedure to obtain repeatable samples at known quality and concentration in the field.

\section{MATERIALS AND METHODS}

\subsection{The monitoring network}

The monitoring network consists of 8 electronic noses equipped with 6 MOS sensors belonging to the "EOS Ambiente" series produced by Sacmi [6]. The instruments were customized in order to make them suitable for the specific application, thereby selecting the sensors to optimize sensitivity towards the typical compounds that are found in oil \& gas plants emissions, and designing a special casement to protect the instruments from the high temperatures that can be reached during summer. 


\subsection{Electronic nose training}

For environmental odour monitoring, a training phase is required to "teach" the electronic nose to recognize the olfactory patterns of the odours it will be exposed to once installed in the field [7]. Because of the huge number of odour sources that are present in the plant to be monitored, it was decided to perform a preliminary study prior to training in order to evaluate how to identify and "group" those number of sources into a limited number of homogeneous olfactory classes to be considered for odour classification:

- "Hydrocarbons" representing the odour caused by the emission of the odorous volatile organic compounds (e.g., aromatics) contained in the crude oil extracted and separated at the monitored plant. Since dealing with an oil extraction and separation facility, and not with a refinery, only one type of crude oil is treated there.

- "Sulphur compounds" represents the odour caused by sulphur compounds that are typically contained mainly in oilfield water but also in the crude oil at low concentrations, such as $\mathrm{H}_{2} \mathrm{~S}$ and mercaptans, which have low odour detection thresholds.

\section{PROCEDURE FOR THE OBTAINMENT OF ODOUR SAMPLES AT KNOWN AND REPEATABLE CONCENTRATION IN THE FIELD}

\subsection{General principles}

The aim was to prepare odour samples at known and repeatable concentration directly in the field, where the typical laboratory equipment is not available. The developed procedure consists of two steps: a first step for the preparation of "concentrated" samples from liquid samples, and a second step for the obtainment of "diluted" samples to be used for the electronic nose performance testing.

\subsection{First step: preparation of concentrated samples}

The odour samples relevant to the olfactory class Hydrocarbons ("HC") were obtained from the headspace of $10 \mu \mathrm{l}$ of liquid crude oil withdrawn from the bottle containing the crude oil coming from the monitored plant by means of a Kartell Pluriel PL10 calibrated pipette (Fig. 1).

The odour samples relevant to the olfactory class Sulphur compounds ("SC") were obtained from the headspace of 1.5 $\mathrm{ml}$ of liquid oilfield water withdrawn from the bottle containing the oilfield water by means of a glass pipette.

Once the liquid quantity $(10 \mu \mathrm{l}$ of oil and $1.5 \mathrm{ml}$ of water, respectively) were withdrawn from the bottle of liquid, this quantity was transferred inside a $6 \mathrm{~L}$-volume Nalophan ${ }^{\mathrm{TM}}$ bag for olfactometric sampling. Finally, the bag was filled with neutral air from a synthetic air bottle. The sample was then kept for 20 minutes at ambient temperature as to achieve equilibrium conditions in the headspace. This procedure was chosen as it allows to have a minimum consumption of liquid, and it was proven to give repeatable results under controlled conditions.

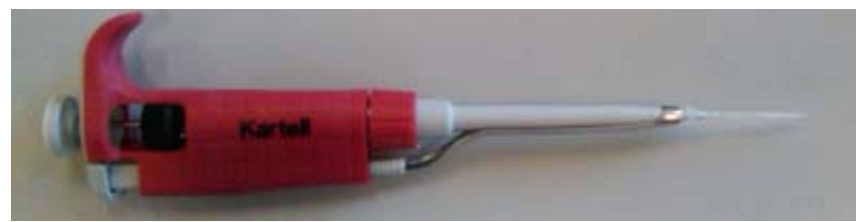

Figure 1. The Kartell Pluriel PL10 pipette

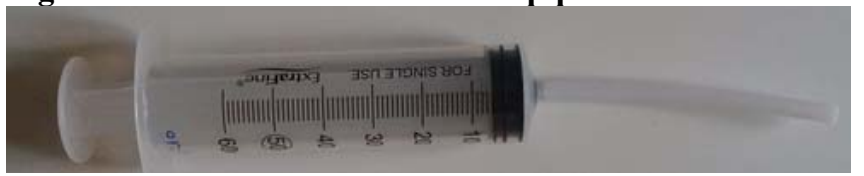

Figure 2. The plastic syringe for gas withdrawal

The samples prepared this way were analysed by dynamic olfactometry [3] to determine their odour concentration, in order to evaluate the dilution ratio required to obtain diluted samples at low odour concentration $\left(\mathrm{c}_{\mathrm{od}}\right)$ values. Four "concentrated" samples for each odour class ("HC" and "SC") were analysed, and they all turned out to have a $\mathrm{c}_{\mathrm{od}}$ of about $1000 \mathrm{ou}_{\mathrm{E}} / \mathrm{m}^{3} \pm 50 \%$, which is the typical uncertainty esteemed for olfactometric measurements [8].

\subsection{Second step: preparation of diluted samples}

The diluted samples both of "HC" and "SC" odours were prepared by withdrawing $25 \mathrm{ml}$ of odorous gas by means of a plastic syringe (Fig. 2) from the concentrated samples prepared according to 3.2 , and transferring this quantity to another 6L Nalophan ${ }^{\mathrm{TM}}$ sampling bag. The plastic syringe is equipped with a Teflon ${ }^{\mathrm{TM}}$ tube having an external diameter of $6 \mathrm{~mm}$ that can be inserted in the sampling bag inlet tube. Two dedicated plastic syringes were used for this operation: one for the "HC" and one for the "SC" odour, respectively, to avoid cross-contamination. After addition of this small quantity of odorous gas from the concentrated sample having a $c_{o d}$ of about $1000 \mathrm{ou}_{\mathrm{E}} / \mathrm{m}^{3}$, the bag (6L) is filled with neutral air from a synthetic air bottle. This corresponds to a dilution of $25 \mathrm{ml}$ in $6000 \mathrm{ml}$, which corresponds to a dilution ratio of $1: 240$, i.e. to a $c_{\text {od }}$ of about $4 \mathrm{ou}_{\mathrm{E}} / \mathrm{m}^{3}$. For the electronic nose performance testing in the field, besides those diluted samples (named " 25 ml") also a few less diluted samples were prepared with a double and fourfold concentration, in order to evaluate the instrument capability to correctly classify odours also in presence of odour peaks. These samples were prepared by transferring 50 and $100 \mathrm{ml}$ of odorous gas from the concentrated sample to a new 6-L bag, respectively, thus obtaining samples with $c_{\text {od }}$ of 8 and $17 \mathrm{ou}_{\mathrm{E}} / \mathrm{m}^{3}$, respectively. Such low $\mathrm{c}_{\text {od }}$ values cannot be measured directly by dynamic olfactometry due to the intrinsic lower detection limit of this technique, but they are evaluated based on the dilution ratios applied to the samples.

\section{RESULTS OF THE ELECTRONIC NOSE NETWORK PERFORMANCE TESTING}

The performance testing of the electronic nose network was carried out in 3 consecutive days, with diluted samples of "HC" and "SC" odours prepared according to the procedure described in 3.3. During these 3 days, different samples 
were analysed by the 8 electronic noses in rotation. The classification provided by the instrument (i.e. the olfactory class attributed to the analysed sample) 10 minutes after connection of the sample to the instrument was considered for performance evaluation, together with the time after which the response becomes stable. After this interval the sample was removed and the recovery time, i.e. the time required to classify the analysed air as neutral air ("AIR") again, was evaluated as well. Tab. 1 is a synthetic table of the outcomes of the performance testing for classification accuracy at low $c_{\text {od }}$ values relevant to the 8 electronic noses that constitute the monitoring network. For each test round, Tab. 1 reports the olfactory class of the analysed sample ("real") and the classification result operated by the electronic nose ("attr."). For a more immediate visualization, correct classifications are reported in green, whereas incorrect classification are in red, italic characters. The last column of Tab. 1 reports the classification accuracy calculated percent ratio between correct classifications and total number of classifications. Based on these results, it is possible to make some considerations:

- The EOS 2 misclassified the "HC" samples at higher $\mathrm{c}_{\mathrm{od}}$ (" 50 ", i.e. $8 \mathrm{ou}_{\mathrm{E}} / \mathrm{m}^{3}$ ), whereas it classified correctly the more diluted ones (" 25 ", i.e. $4 \mathrm{ou}_{\mathrm{E}} / \mathrm{m}^{3}$ ), giving an overall classification accuracy of $80 \%$, which could presumably be further improved by optimizing the training set.

- The EOS 4 misclassified the diluted sample of "SC" (" 25 ", i.e. $4 \mathrm{ou}_{\mathrm{E}} / \mathrm{m}^{3}$ ) on the first day. This result might have been affected negatively by the fact that the corresponding test was carried out at the end of the day, using a sample that had been prepared over 10 hours before the analysis, which might have been therefore depleted in its odour content. This impression seems confirmed by the fact that on the next days the new sample of "SC" at low c cod was classified correctly.

- The other 6 instruments gave an overall classification accuracy of $100 \%$.

As a critical discussion, it is important to highlight that there is a significant difference between samples with liquid headspace injections and real atmospheres, the latter being less reproducible. This procedure was chosen as a reasonable compromise for repeatable performance testing with samples of sufficient representativeness in the field.
The developed procedure for the obtainment of odour samples at known and repeatable concentration proved to be effective for electronic nose performance testing directly in the field. All instruments turned out to have an accuracy of above $80 \%$ towards the tested odour types, although it must be kept into mind that there is still a difference between the tested samples obtained liquid headspace injections and real atmospheres. This result is nonetheless particularly satisfying in consideration of the low $c_{o d}$ values (close to the odour detection threshold) of the tested samples.

\section{REFERENCES}

[1] M. Brancher, K.D. Griffiths, D. Franco, and H. de Melo Lisboa, "A review of odour impact criteria in selected countries around the world", Chemosphere 168, pp. 15311570, 2017.

[2] L. Capelli, S. Sironi, and R. Del Rosso, "Electronic Noses for Environmental Monitoring Applications", Sensors 14, pp. 19979-20007, 2014.

[3] CEN, EN 13725:2003. Air Quality-Determination of Odour Concentration by Dynamic Olfactometry, Comitée Européen de Normalisation, Brussels, 2007.

[4] L. Capelli, L. Dentoni, S. Sironi, and R. Del Rosso, "The need for electronic noses for environmental odour exposure assessment", Water Science and Technology 69, pp. 135141, 2014.

[5] B. Milan, S. Bootsma, and I. Bilsen, "Advances in odour monitoring with E-Noses in the Port of Rotterdam" Chemical Engineering Transactions 30, pp. 145-150, 2012.

[6] L. Dentoni, L. Capelli, S. Sironi, R. Del Rosso, S. Zanetti, and M. Della Torre, "Development of an Electronic Nose for Environmental Odour Monitoring” Sensors 12, pp. 14363-14381, 2012.

[7] L. Capelli, S. Sironi, P. Céntola, R. Del Rosso, and M. Il Grande, "Electronic noses for the continuous monitoring of odours from a wastewater treatment plant at specific receptors: Focus on training methods", Sensors and Actuators B, Chemical 131, pp. 53-62, 2008.

[8] J.V. Klarenbeek, N.W.M. Ogink, and H. Van der Voet, "Odor measurements according to EN 13725: A statistical analysis of variance components", Atmospheric Environment 86, pp. 9-15, 2014.

\section{CONCLUSIONS}

Table 1. Synthetic table of the electronic nose network performance testing results

\begin{tabular}{|c|c|c|c|c|c|c|c|c|c|c|c|c|c|c|c|c|c|c|c|c|c|}
\hline \multirow{2}{*}{ E-Nose } & \multicolumn{2}{|c|}{ Test ro. 1} & \multicolumn{2}{|c|}{ Test ro. 2} & \multicolumn{2}{|c|}{ Test ro. 3} & \multicolumn{2}{|c|}{ Test ro. 4} & \multicolumn{2}{|c|}{ Test ro. 5} & \multicolumn{2}{|c|}{ Test ro. 6} & \multicolumn{2}{|c|}{ Test ro. 7} & \multicolumn{2}{|c|}{ Test ro. 8} & \multicolumn{2}{|c|}{ Test ro. 9} & \multicolumn{2}{|c|}{ Test ro. 10} & \multirow{2}{*}{ Accuracy } \\
\hline & real & attr. & real & attr. & real & attr. & real & attr. & real & attr. & real & attr. & real & attr. & real & attr. & real & attr. & real & attr. & \\
\hline EOS & HC 25 & $\mathrm{AC}$ & 25 & C & 50 & IC & & & $\mathbf{R}$ & R & 5 & $\mathrm{HC}$ & & C & 50 & SC & 0 & C & IR & IR & \\
\hline 52 & 5 & SC & 5 & IC & 0 & $S C$ & & SC & & IR & & UN & & $\mathrm{HC}$ & & SC & & SC & $\mathbf{R}$ & AIR & \\
\hline EOS 3 & C 25 & IC & 5 & SC & 0 & $\mathrm{HC}$ & & & & AIR & 100 & SC & & & & & & & IR & AIR & $00 \%$ \\
\hline EOS 4 & C 25 & $\mathrm{HC}$ & 25 & $H C$ & SC 50 & $\mathrm{SC}$ & & & $\mathbf{R}$ & AIR & SC 25 & SC & SC $1 C$ & SC & & & & & AIR & AIR & $83 \%$ \\
\hline EOS 5 & C 25 & $\mathrm{HC}$ & C 25 & SC & HC 50 & $\mathrm{HC}$ & & & AIR & AIR & SC 100 & SC & & & & 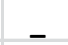 & & _ & AIR & AIR & $100 \%$ \\
\hline EOS 6 & SC 25 & SC & C 50 & $\mathrm{HC}$ & HC 25 & $\mathrm{HC}$ & & - & IR & AIR & SC 100 & SC & & & & - & & - & AIR & AIR & $\%$ \\
\hline EOS 7 & C 25 & $\mathrm{HC}$ & $\mathbf{2} 25$ & SC & HC 50 & $\mathrm{HC}$ & SC 50 & SC & $A$ & AIR & HC 25 & $\mathrm{HC}$ & SC 25 & SC & SC 50 & SC & SC 100 & SC & AIR & AIR & $100 \%$ \\
\hline EOS 8 & C 25 & C & C 25 & C & C 75 & SC & & & AIR & AIR & SC 100 & SC & & & & & - & & AIR & AIR & $100 \%$ \\
\hline
\end{tabular}

